\title{
True time reversal via dynamic Brillouin gratings in polarization maintaining fibers
}

\author{
Sanghoon Chin, Nikolay Primerov, Kwang Yong Song, Luc Thévenaz \\ Ecole Polytechnique Fédérale de Lausanne, STI-GR-SCI Station 11, CH-1015 Lausanne, Switzerland \\ luc.thevenaz@epfl.ch \\ Marco Santagiustina, Leonora Ursini \\ CNIT-Department of Information Engineering, University of Padova, via Gradenigo 6b, 35131, Padova, Italy \\ marco.santagiustina@unipd.it
}

\begin{abstract}
A novel technique to realize true time reversal of an optical signal, using dynamic Brillouin gratings in high-birefringence fibers, is proposed. A data sequence of optical pulses with 2-ns duration was efficiently time-reversed.

(C) 2010 Optical Society of America

OCIS codes: (060.4370) Nonlinear optics, fibers; (290.5900) Scattering, stimulated Brillouin; (070.4340) Nonlinear optical signal processing
\end{abstract}

In acoustics and electromagnetics, low frequency time reversal has been successfully realized, showing many potential applications in communications, medicine and material analysis $[1,2,3]$. Experimental demonstration of ultrafast optical waveform time reversal has been also made, using various physical phenomena such as spectrally decomposed-wave mixing [4, 5], photon echo [6] and spectral hole-burning holography [7]. In this paper, we propose and experimentally demonstrate a novel technique to perform true time reversal (TTR) of optical signals in an all-fiber configuration making use of dynamic Brillouin gratings (DBG) in high birefringence optical fibers [8]. The proposed setup is simpler with respect to the fiber optic time-lens proposed in [9], and so very attractive for applications in optical communications and microwave-photonics [10]. Light storage and retrieval in optical fibers has been succesfully demonstrated $[11,12]$ by exploiting the stimulated Brillouin scattering (SBS) occurring between two counter-propagating optical pulses. The acoustic wave, generated by the interaction, retains the characteristics of the colliding pulses and actually modulates the fiber refractive index, thus creating a DBG (Bragg-like). A new light beam can be scattered on to the DBG, thus enabling the original optical waveform to be retrieved [11,12]. In polarization maintaining (PM) fibers, the storage/retrieval processes can be realized on the two orthogonal states of polarization aligned to the birefringence axis $[8,13]$ thus enabling a practical method to decouple the read and write channels. In fact, the acoustic wave equally scatters all light polarizations owing to its longitudinal nature. In Fig. 1, the process to achieve TTR is sketched. The linearly polarized input data sequence, $A_{d}^{i n}$, and the counter-propagating (write) pulse, $A_{w}$, are launched along the slow axis of a PM fiber. The signals interact through SBS if their frequencies satisfy the Brillouin condition $v_{d}^{i n}-v_{w}=v_{B}$. The interaction generates an acoustic wave, $Q$, in which the data sequence $A_{d}^{i n}$ is spatially stored. The reading pulse, $A_{r}$, linearly polarized along the fast axis is launched just after the data sequence, thus interacting with the stored acoustic wave and generating a counter-propagating output waveform, $A_{d}^{\text {out }}$, polarized along the fast axis and at frequency $v_{d}^{\text {out }}=v_{r}-v_{B}$. The part of the input sequence which is stored last is the first to be retrieved and so TTR is realized. The write/input data and read/output data pulses also satisfy SBS phase matching conditions from which one obtains $v_{r}=\left(1+\Delta n / n_{s}\right) v_{d}^{i n}$, where $\Delta n=n_{s}-n_{f}$ is the fiber birefringence $[8,13]$.

The process is governed by the following equations:

$$
\begin{array}{rlrl}
\partial_{z} A_{d}^{\text {in }}+\beta_{1 s} \partial_{t} A_{d}^{\text {in }} & =-\eta_{1} g_{B} Q A_{w}+j \eta_{2} \gamma\left(\left|A_{d}^{\text {in }}\right|^{2}\right) A_{d}^{\text {in }}, & -\partial_{z} A_{w}+\beta_{1 s} \partial_{t} A_{w}=\eta_{1} g_{B} Q^{*} A_{d}^{\text {in }}+j \eta_{2} \gamma\left(\left|A_{w}\right|^{2}\right) A_{w}, \\
\partial_{z} A_{r}+\beta_{1 f} \partial_{t} A_{r} & =-\eta_{1} g_{B} Q A_{d}^{\text {out }}+j \eta_{2} \gamma\left(\left|A_{r}\right|^{2}\right) A_{r}, & -\partial_{z} A_{d}^{\text {out }}+\beta_{1 f} \partial_{t} A_{d}^{\text {out }}=\eta_{1} g_{B} Q^{*} A_{r}+j \eta_{2} \gamma\left(\left|A_{d}^{\text {out }}\right|^{2}\right) A_{d}^{\text {out }}, \\
2 \tau_{B} \partial_{t} Q+Q & =A_{d} A_{w}^{*}+\left(A_{d}^{\text {out }}\right)^{*} A_{r} .
\end{array}
$$

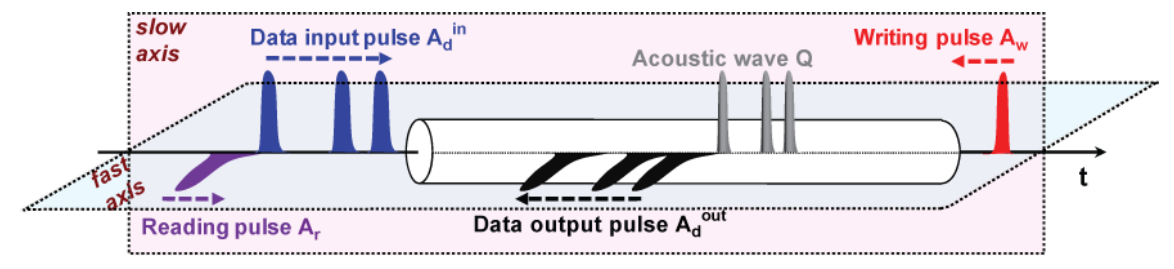

Fig. 1: Process for the realization of TTR in PM fibers via DBG. 


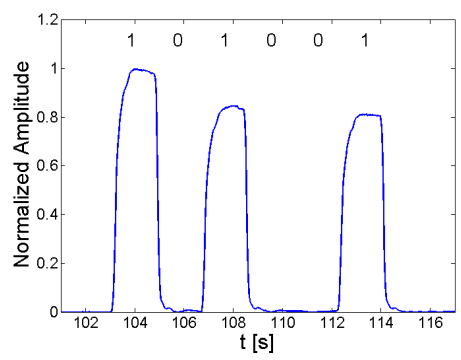

Fig. 2: Input data sequence, composed by 6 bits of duration $2 \mathrm{~ns}$.

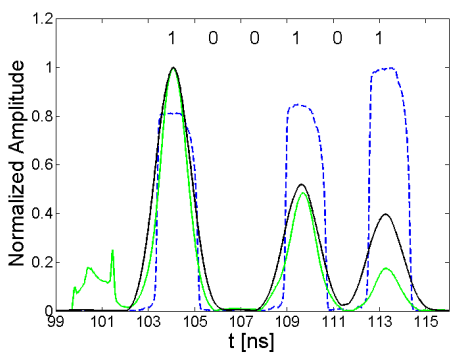

Fig. 3: Comparison of experimentally (green curve) and numerically (black curve) reversed sequence with ideally reversed input sequence (blue curve). Write/read pulses of 2 ns duration.

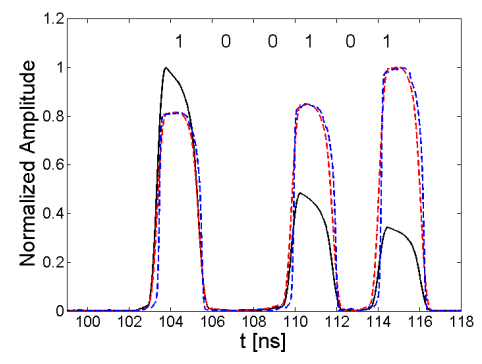

Fig. 4: Comparison of simulation results without (black curve) and with (red curve) the exponential post-correction (write/read pulses of $0.25 \mathrm{~ns}$ duration) with ideally reversed input sequence (blue curve).

The main difference with eqs. of ref. [13] is that the self phase modulation is taken into account because of the high peak power $(>100 \mathrm{~W})$ of the write/read pulses. The parameters of the simulations and experiments implementing the scheme of Fig. 1 are: fiber length $L=20 \mathrm{~m}$, input data central wavelength $\lambda_{d}=1535 \mathrm{~nm}$, fiber birefringence $\Delta n=5 \cdot 10^{-4}$, SBS shift $v_{B}=10.93 \mathrm{GHz}$, SBS gain $g_{B}=5 \cdot 10^{-11} \mathrm{~m} / \mathrm{W}$, acoustic wave lifetime $\tau_{B}=4 n s$, nonlinearity coefficient $\gamma=2.6 \cdot 10^{-3} \mathrm{~m}^{-1} W^{-1}$. $\eta_{1}=2 \cdot 10^{-3} \Omega^{-1}$ and $\eta_{2}=8 \cdot 10^{-14} \Omega^{-1} m^{2}$ are normalization factors. Peak powers are: $P_{d}^{i n}=500 \mathrm{~mW}, P_{w}=160 \mathrm{~W}, P_{r}=100 \mathrm{~W}$ respectively for the data, the write and read pulses. Fig. 2 shows the input data sequence of 6 bits with duration $2 \mathrm{~ns}$ each. The write and read pulses in the experiments have the same duration. In Fig. 3, TTR is experimentally demonstrated (green curve); the agreement with the numerical results (black curve) obtained integrating the governing eqs. with the input sequence of Fig. 2 is good. However, output pulses are broader and weaker of those of an ideally reversed sequence (blue curve). The differences in the peak levels are mainly due to the fact that the acoustic wave decays exponentially $\left(\exp \left[-t /\left(2 \tau_{B}\right)\right]\right)$ during storage. So, the first bit to be stored, i.e. the last to be retrieved, is affected by a larger decay. The broadening, instead, is due to the inherent spectral filtering effect during write/read process that realizes a convolution of the optical pulses, as can be demonstrated by direct integration of eq. 3. The explanations of the observed experimental distortions are confirmed in Fig. 4, where we show the results of the numerical integration of eqs. $(1,2,3)$ with the input sequence of Fig. 2 but by numerically post-compensating the exponential decay and by using shorter write/read pulses $(0.25 \mathrm{~ns})$ (black curve).

In conclusion, a novel method for true time reversal has been proposed by exploiting DBG in PM fibers. A data sequence of $2 \mathrm{~ns}$ optical pulses has been experimentally time reversed. The numerical analysis is in good agreement with experiments and demonstrates that high-fidelity reversal can be achieved. Possible applications in electromagnetics [2] and MWP [10] can be envisaged.

This research has been carried out within the FP7, FET-Open, Project GOSPEL, n. 219299.

\section{References}

[1] M. Fink, "Time-reversed acoustics," Physics Today, 30, 34-40 (1997).

[2] G. Lerosey, J. de Rosny, A. Tourin, A. Derode, G. Montaldo and M. Fink, ’Time Reversal of Electromagnetic waves," Phys. Rev. Lett., 92, 193904 (2005).

[3] H. T. Nguyen, J. B. Andersen and G. F. Pedersen, "The Potential Use of Time Reversal Techniques in Multiple Element Antenna Systems," IEEE Commun. Lett., 9, 40-42 (2005).

[4] D. A. B. Miller, ’Time reversal of optical pulses by four-wave mixing," Opt. Lett., 5, 300-302 (1980).

[5] D. Marom, D. Panasenko, R. Rokitski, P-C. Sun and Y. Fainman, "Time reversal of ultrafast waveforms by wave mixing of spectrally decomposed waves," Opt. Lett., 25, 132-134 (2000).

[6] N. W. Carlson, L. J. Rothberg, A. G. Yodh, W. R. Babbitt and T. W. Mossberg, "Storage and time reversal of light pulses using photon echoes," Opt. Lett., 8, 483-485 (1983).

[7] A. Rebane , J. Aaviksoo and J. Kuhl, "Storage and time reversal of femtosecond light signals via persistent spectral hole burning holography," Appl. Phys. Lett., 54, 93-95 (1989).

[8] K. Y. Song, W. Zou, Z. He and K. Hotate, "All-optical dynamic grating generation based on Brillouin scattering in polarization maintaining fiber," Opt. Lett., 33, 926-928 (2008).

[9] F. Coppinger, A.S. Bhushan and B. Jalali, "Time reversal of broadband microwave signals," Elect. Lett., 35, 1230-1232 (1999).

[10] J. Capmany, D. Novak, "Microwave photonics combines two worlds," Nature Photonics 1, 319-322 (2007).

[11] Z. Zhu, D. J. Gauthier and R. W. Boyd, ”Stored light in an optical fiber via stimulated Brillouin scattering," Science, 318, 1748-1750 (2008).

[12] Y.Cao, P. Lu, Z. Yang, W. Chen, ”An efficient method of all-optical buffering with ultra-small core photonic crystal fibers," Opt. Expr., 16, $14142-14150$ (2008).

[13] V.P. Kalosha, W. Li, F. Wang, L. Chen, X. Bao, "Frequency-shifted light storage via stimulated Brillouin scattering in optical fibers," Opt. Lett., $33,2048-2050$ (2008) 